Orange Journal / Volumen 2 Número 4/ Julio - diciembre 2020

DOI: https://doi.org/10.46502/issn.2710-995X/2020.4.01

\title{
Usos etnofarmacológicos de plantas en el tratamiento de enfermedades crónicas no transmisibles en Santiago de Cuba
}

\author{
Ethnopharmacological use of plants in the treatment of chronic \\ non-communicable diseases in Santiago de Cuba
}

Recibido: 12 de diciembre de 2020

Aceptado: 5 de abril de 2021

Escrito por:

Rut Benita Yero Haber ${ }^{1}$

Julio César Escalona Arranz ${ }^{2}$

https://orcid.org/0000-0003-3609-4451

\section{Resumen}

Existe una marcada tendencia en la utilización empírica de plantas medicinales para el tratamiento de enfermedades crónicas no transmisibles siendo necesario el inventario de las mismas. En el presente trabajo se realizó un estudio etnofarmacológico, exploratorio y descriptivo, con enfoque cualitativo y cuantitativo en comunidades urbanas y rurales de Santiago de Cuba. Se entrevistaron un total de 100 pacientes, realizando la caracterización sociodemográfica y clínica de la comunidad; el análisis de las variables relativas al uso de plantas medicinales y la selección de especies vegetales con potencialidades para el desarrollo de estudios fitoquímicos y farmacológicos. Los entrevistados refirieron las especies más empleadas para el tratamiento de sus enfermedades crónicas, prevaleciendo el sexo femenino, el grupo de edades comprendido entre 40 y 59 años de edad y el nivel de escolaridad en la enseñanza técnico-profesional. Se informaron un total de 51 especies, agrupadas 34 familias. Las plantas con mayores niveles de uso significativo, índice de valor de uso e índice de fidelidad fueron, en el municipio Santiago de Cuba: Allium sativum L. y Ocimum gratissimum (L.) ( $\mathrm{IF}=96 \%$ ), Chamaemelum nobile L. y Eucalyptus maculata ( $\mathrm{IF}=90 \%$ ), mientras que en Songo La Maya: Calendula officinalis (L.), Carica papaya (L.) $(\mathrm{IF}=100 \%)$ y Citrus reticulate ( $\mathrm{IF}=95.65 \%)$. Las especies con mayores potencialidades para futuros estudios fitoquímicos y farmacológicos resultaron ser: Eucalyptus maculata, Camellia sinensis, Ocimum gratissimum L, Psidium guajaba L, Persea americana, Anacardium occidentale L., Nasturtiumo fficinale R. Br, Zingiber officinale, Citrus paradise, Plectranthus neochillus Benth ex E. Meg y Dichrostachys cinerea (L.) Wight \&Arn.

Palabras clave: asma bronquial, diabetes mellitus, etnofarmacología, hipertensión arterial, plantas medicinales.

\footnotetext{
${ }^{1}$ Estudiante de cuarto año Lic. Ciencias Farmacéuticas. Miembro del Grupo Científico-estudiantil FARMACIA Y COMUNIDAD. Departamento de Farmacia. Facultad de Ciencias Naturales y Exactas. Universidad de Oriente. Santiago de Cuba. Cuba.

${ }^{2}$ Doctor en Ciencias Farmacéuticas. Profesor Titular. Departamento de Farmacia. Facultad de Ciencias Naturales y Exactas. Universidad de Oriente. Santiago de Cuba. Cuba.
} 


\begin{abstract}
There is a marked trend in the empirical use of medicinal plants for the treatment of chronic non-communicable diseases, requiring an inventory of them. In the present work, an ethnopharmacological, exploratory and descriptive study was carried out, with a qualitative and quantitative approach in urban and rural communities of Santiago de Cuba. With this purpose, residents of Santiago de Cuba and Songo la Maya municipalities were interviewed, carrying out the sociodemographic and clinical characterization of the community. It was also analyzed variables related to the use of medicinal plants and the selection of plant species with potential for the development of phytochemical and pharmacological studies. A total of 100 patients were interviewed with female sex prevalence, age group between 40 and 59 years old and technical-professional educational level. A total of 51 species were reported, grouped 34 families. The plants with the highest levels of: "significant use", "value index" and "fidelity index" were, Allium sativum L and Ocimum gratissimum (L.) (IF = 96\%), Chamaemelum nobile L and Eucalyptus maculata $(\mathrm{IF}=90 \%$ ) for Santiago de Cuba municipality, while for Songo La Maya were Calendula officinalis (L.), Carica papaya (L.) (IF = 99\%) and Citrus reticulate (IF = 95.65\%). The species that classifies with the greatest potential for future phytochemical and pharmacological studies were: Eucalyptus maculata, Camellia sinensis, Ocimum gratissimum L, Psidium guajaba L, Persea americana, Anacardium occidentale L., Nasturtium officinale R. Br, Zingiber officinale, Citrus paradise, Plectranthus neochillus Benth ex E. Meg y Dichrostachys cinerea (L.) Wight \&Arn.
\end{abstract}

Keywords: bronchial asthma, diabetes mellitus, ethnopharmacology, high blood pressure, medicinal plants.

\title{
Introducción
}

Desde los tiempos más remotos se han empleado las plantas con fines curativos. Sin embargo, su uso fue relegado a un segundo plano a medida que la ciencia fue desarrollando medicamentos sintéticos, hasta que en las últimas décadas se ha experimentado internacionalmente un retorno hacia el uso de la medicina natural en las terapias de diferentes patologías (Badke et al, 2012).

Las enfermedades crónicas no transmisibles tienden a ser de larga duración y resultan de la combinación de factores genéticos, fisiológicos, ambientales y conductuales (Mora et al., 2017). La OMS señala que el $80 \%$ de la población mundial utiliza las plantas como principal remedio medicinal, práctica asociada al empirismo en muchos casos, ya que son insuficientes los estudios químicos, clínicos y epidemiológicos que confirmen los efectos fisiológicos de las plantas y los principios activos responsables (Escalona et al., 2015). Dicha utilización, no asegura la eficacia y validez que se requiere para la aplicación terapéutica de especies vegetales en el tratamiento de las enfermedades crónicas no transmisibles, puesto que puede provocar la aparición de reacciones adversas que lejos de contribuir al bienestar del paciente, pueden empeorar su cuadro clínico. Los estudios etnobotánicos no solo validan la posible utilización o no de un producto natural, sino que constituyen una nueva ventana hacia la selección de las plantas medicinales con potencialidades para el desarrollo de estudios fitoquímicos y farmacológicos que 
permitan la búsqueda de nuevas sustancias con propiedades terapéuticas. Considerando lo anteriormente expuesto se define como objetivo de investigación la caracterización del uso tradicional de plantas medicinales para el tratamiento de las enfermedades crónicas no transmisibles por la población de las comunidades: Centro Urbano "Sierra Maestra" y "Alto Songo". Para ello se determinaron las plantas de mayor uso popular en las comunidades describiendo las formas de consumo, sus partes más empleadas, la fuente de obtención, así como conocimiento de su uso. Finalmente se seleccionaron las especies vegetales con potencialidades para el desarrollo de estudios fitoquímicos $y$ farmacológicos.

\section{Marco Teórico}

Las Enfermedades Crónicas No Transmisibles (ECNT) son enfermedades de larga duración cuya evolución es generalmente lenta. Estas enfermedades representan una verdadera epidemia que va en aumento debido al envejecimiento de la población y los modos de vida actuales que acentúan el sedentarismo y la mala alimentación. En su conjunto son responsables de más del $60 \%$ de las muertes, $80 \%$ de las cuales ocurren en países de bajos y medianos ingresos (Chávez et al., 2016). Las principales ECNT son la diabetes, las enfermedades cardiovasculares, el cáncer, las enfermedades respiratorias crónicas y la enfermedad renal, y se caracterizan por compartir los mismos factores de riesgo. La mayor parte de las ECNT pueden atribuirse a la presencia de un número reducido de factores de riesgo (FR), algunos de los cuales son evitables. Entre los FR evitables más vinculados figuran el consumo de tabaco, el consumo excesivo de alcohol, una dieta inadecuada, insuficiente actividad física, altos niveles de presión arterial, colesterol y glucosa, sobrepeso y obesidad. En Cuba, las ECNT se encuentran dentro de las principales causas de muerte, de lo que puede inferirse la prioridad que tiene la investigación epidemiológica en la identificación de los factores de riesgo de las ECNT y su reducción (Rodríguez y García, 2018).

La hipertensión arterial (HTA) es la más común de las enfermedades crónicas no transmisibles, caracterizada por un incremento continuo de las cifras de la presión sanguínea en las arterias, representa por sí una enfermedad y constituye un factor de riesgo para otras enfermedades. Esta enfermedad se asocia a tasas de morbilidad y mortalidad considerablemente elevadas, por lo que se considera uno de los problemas más importantes de salud pública (Soca et al., 2017). Es la afección crónica más frecuente en la población adulta del planeta, se comporta como factor de riesgo para padecer las enfermedades que se encuentran entre las más importantes causas de muerte en los países desarrollados y en la mayor parte de los países en vías de desarrollo.

Existen diferentes grupos de fármacos que son utilizados para el tratamiento de este padecimiento como es el caso de los diuréticos (espirinolactona, furosemida, hodroclorotiazida), los antagonistas de los canales de calcio (nifedipino, amlodipino), los betabloqueadores (atenolol, propranolol), los inhibidores de la enzima convertidora de angiotensina (enalapril, captopril), los simpaticolíticos (metildopa) y los ARA II (losartán). Con frecuencia, se usan uno o más de estos medicamentos para tratar la hipertensión arterial (Herrera, 2017). 
La diabetes es una enfermedad crónica en la cual el cuerpo no puede regular la cantidad de azúcar en la sangre. La diabetes tipo 1 aparece frecuentemente en la infancia, la adolescencia y los primeros años de la vida adulta. Acostumbra a presentarse de forma brusca y muchas veces independientemente de que existan antecedentes familiares. Las causas de la diabetes tipo 1 son principalmente la destrucción progresiva de las células del páncreas, que producen insulina. La diabetes tipo 2 surge generalmente en edades más avanzadas. Se origina debido a una producción de insulina escasa, junto con el aprovechamiento insuficiente de dicha sustancia por parte de las células. El tratamiento de la diabetes mellitus se basa en tres pilares: dieta, ejercicio físico y medicación. Tiene como objetivo mantener los niveles de glucosa en sangre dentro de la normalidad para minimizar el riesgo de complicaciones asociadas a la enfermedad. Los fármacos hipoglucemiantes orales se prescriben a pacientes con diabetes tipo 2 que no consiguen descender la glucemia a través de la dieta y la actividad física. El tratamiento con insulina es indicado en pacientes con diabetes tipo 1. Es necesaria la administración exógena de insulina, ya que el páncreas es incapaz de producir esta hormona. Las necesidades de insulina varían en función de los alimentos que se ingieren y de la actividad física que se realiza (Hashmi \& Khan, 2016).

El asma es una enfermedad del sistema respiratorio caracterizada por una inflamación crónica de la vía aérea, cuyas manifestaciones clínicas son heterogéneas y variables en el tiempo y consisten en sibilancias, dificultad respiratoria, opresión torácica y tos. El origen de la enfermedad es complejo e involucra la inflamación, la obstrucción intermitente y la hiperreactividad de las vías respiratorias. El tratamiento convencional del asma bronquial puede ser sintomático cuando tiene por objeto interrumpir la crisis mediante medicamentos de acción rápida, como la adrenalina, corticoides, oxigenoterapia, y preventivo cuando se indica el uso regular de broncodilatadores, antihistamínicos, corticosteroides, terapia respiratoria e inmunoterapia específica (Agarwal et al, 2015).

La etnobotánica estudia las relaciones entre los grupos humanos y su entorno vegetal, es decir, el uso y aprovechamiento de las plantas en los diferentes espacios culturales y en el tiempo. Dentro de este campo se desarrollan los estudios etnofarmacológicos, los cuales abarcan el uso tradicional y los efectos de las sustancias naturales dotadas de actividad biológica (tanto plantas como animales y minerales medicinales). En Cuba, la introducción de la Medicina Tradicional comienza en el siglo XV. Primero, introducida por la colonia española, la cual tenía una enorme influencia de la medicina oriental (Fernández \& Armas, 2013). En 1968 se realizan en Cuba los primeros trabajos sobre los usos de las plantas medicinales por especialistas de la Academia de Ciencias de Cuba. Estos pretendían, a través de una "Comisión de Plantas Medicinales", promover el cultivo y la explotación de las plantas aromáticas y medicinales, para así llegar a desarrollar una nueva industria en el país, con la finalidad de crear nuevas fuentes de trabajo (Pérez et al., 2011). Por esta fecha aparecen los trabajos del Dr. Juan Tomás Roig y Mesa. Este, en sus obras, identificó 595 especies de plantas medicinales empleadas por la población cubana para diferentes usos curativos (Roig, 1974).

Durante el año 2011 se publicó una investigación centrada en las propiedades diuréticas de las plantas, se registraron 20 especies, 8 plantas con un mayor nivel de uso y 12 de las plantas reportadas como diuréticas carecían de validación científica. El estudio se realizó 
en 10 municipios pertenecientes a la provincia de Villa Clara, entre los cuales se encuentra el municipio de Quemado de Güines, el cual fue el centro de estudio para otra investigación publicada en el mismo año, con la participación de muchos de los autores de la anterior y centrada de la misma manera en las plantas utilizadas como diuréticos. En este estudio se identificaron 26 especies medicinales, 10 con un mayor nivel de uso significativo y 14 carecían de validación a nivel experimental en Cuba, las plantas reportadas coinciden con las de la investigación anterior agregando 6 plantas utilizadas con este propósito (Pérez et al., 2011).

En el año 2018 se llevó a cabo un estudio etnofarmacológico en siete comunidades de Holguín, región oriental de Cuba. En el mismo se 195 especies, distribuidas en 166 géneros y 70 familias, para el tratamiento de 17 categorías de usos, entre las que resaltaban las afecciones hepáticas y estomacales. Las especies medicinales con mayor valor de uso fueron Lippia alba (Mill.) N.E. Br. ex Britton \& P. Wilson (0.236) y Annona muricata L. (0.194). se lograron altos índices de consenso entre los informantes para el cáncer y los tumores (Heredia et al., 2018). Otras investigaciones por su parte, consideran temas más específicos y centrados en una única especie (Rodríguez et al. 2020).

Cuba cuenta con una gran diversidad biológica. Gran parte de sus poblaciones usan plantas como medicinas, lo que constituye un recurso potencial para afrontar las enfermedades de la población en general. Para esto, se requiere de estudios sistemáticos con inventarios, reconocimientos y validaciones científicas, como parte de las políticas adecuadas para el manejo de este recurso.

\section{Materiales y métodos}

\section{Características generales de la investigación}

Se realizó un estudio exploratorio y descriptivo con un enfoque cualitativo y cuantitativo, aplicando el método etnofarmacológico, en dos localidades de la provincia Santiago de Cuba. Estas localidades fueron la capital provincial (ciudad de Santiago de Cuba) y una zona de carácter rural en el municipio de Songo la Maya. Las encuestas se realizaron durante el período comprendido entre los meses de diciembre de 2018- diciembre de 2019, a pobladores que padecieran de alguna de las siguientes patologías: hipertensión arterial, diabetes mellitus tipo 2 o asma bronquial y que a su vez consumieron plantas medicinales para controlar estas patologías bases. De tal forma se obtendría una caracterización del uso etnofarmacológico actual de plantas medicinales para el tratamiento de las Enfermedades Crónicas No Transmisibles (ECNT).

\section{Muestra de Estudio y recogida de la información}

Para el estudio se utilizó una muestra heterogénea, constituida por personas de ambos sexos y diferentes edades, de variado nivel de escolaridad y ocupación. Se aplicó un muestreo no probabilístico aplicando la técnica de bola de nieve. Por consideraciones éticas, siempre se tuvo en cuenta la voluntariedad y consentimiento de los informantes para participar en el estudio. 
Para la recogida de la información los participantes fueron encuestados utilizando la entrevista semiestructurada y empleando como instrumento el cuestionario que establece el Programa de investigación aplicada a la medicina popular del Caribe (TRAMIL) con algunas modificaciones que responde a los objetivos de la investigación (Intriago et al, 2015).

\section{Recolección e identificación de las plantas}

Las plantas fueron recolectadas en el área de estudio. Se les realizó la identificación taxonómica en el Centro Oriental de Ecosistemas y Biodiversidad (BIOECO), de la provincia de Santiago de Cuba.

\section{Análisis de los datos}

Los datos obtenidos de las encuestas fueron recopilados y organizados en hojas de cálculo de Excel del paquete de Microsoft Office 2016, creada con este propósito. Los mismos permitieron la caracterización de la muestra de estudio y el cálculo de índices muy empleados en estudios etnofarmacológico. Estos se basan en el consenso de los informantes y permiten relacionar el uso de las especies con el conocimiento popular acerca de ellas.

\section{Variables sociodemográficas}

A partir de la información recopilada en las encuestas se realizó la distribución de los pacientes entrevistados según las siguientes variables sociodemográficas:

- Edad: se agrupan en los siguientes grupos etáreos: 16-39 años, 40-59 años y $\geq 60$ años según lo que establece el Programa Nacional de Medicamentos del MINSAP.

- Sexo: Masculino y Femenino

- Nivel de Escolaridad: primaria, secundaria, bachiller, técnico, universitario

- Actividad que realiza: Ama de casa, yerbero/vendedor de plantas, trabajador(a), jubilado(a), curanderos, otra

\section{Determinación de las plantas con mayor uso}

Según los resultados de las encuestas, las plantas fueron clasificadas y agrupadas según familia, nombre científico y vernáculo. Se determinaron las familias más reportadas del total de plantas informadas. Para la denominación del nombre científico se utilizó el Diccionario botánico de nombres vulgares cubanos de Roig (Roig, 1988), Plantas medicinales aromáticas o venenosas de Cuba de Roig (Roig, 1974).

El total de usos reportados por los encuestados, fueron agrupados por categorías, seleccionándose los citados con mayor frecuencia. Se accedió las bases de datos PubMed (en versión gratuita de MEDLINE) y google schoolar; Así como a revistas especializadas tales como:, Journal of Ethnopharmacology, Phytochemistry, British Journal of Phytotherapy, Journal of Natural Products, Chinese Medical Journal, Plantes Medicinales et Phytotherapie, Revista Paulista Medica (Brasil), Revista Cubana de Plantas 
Medicinales, Revista Brasileña de Farmacognosia, y el Boletín Latinoamericano y del Caribe de Plantas Medicinales y Aromáticas (BLACPMA) entre otras. Fue empleado además el Formulario Nacional de Plantas Medicinales.

Descripción de métodos de preparación, partes empleadas, fuente de obtención y conocimiento de su uso.

De las encuestas aplicadas se obtuvo la información referente a los métodos que emplean los encuestados para la preparación de las plantas, las partes más utilizadas, las vías de administración y conocimiento de su uso, declarando como:

- Formas de uso: Planta fresca, seca, indistintamente, otra

- Formas de preparación: Decocción, infusión, maceración, emplasto, amuleto, otra

- Vías de administración: Interna (Oral, sublingual, otra); Tópica (Dérmica, nasal, otra)

- Parte empleada: Hoja, fruto, semilla, raíz, corteza, flores, planta completa, látex, resina.

- En qué estado de la planta la utiliza: floración, fructificación, otra.

- Origen de la planta: cultivada, espontánea, compra a yerberos, farmacia, otra

- Adquisición del conocimiento sobre la(s) planta(s): familia, amigos, vecinos, médicos, medios de difusión, yerberos, curanderos, libros, revistas.

\section{Cálculo de indicadores etnobotánicos cuantitativos}

De todas las plantas informadas por la población encuestada en el área se escogieron las más reportadas con su nombre vulgar, científico y familia. Para estas plantas y partiendo de toda la información recopilada se calcularon los siguientes parámetros:

- Índice de valor de uso (IVU): Para estimar el índice de valor de uso (IVU) de cada especie para todos los informantes se utilizó la fórmula (Luján, 2015):

$$
\text { IVUs }=\sum \text { Uvis/Ns }
$$

Donde: Uvis: número de usos mencionados por cada informante (i) y por cada especie (s); Ns: número de informantes entrevistados.

- Nivel de uso significativo (NUS): Se calcula según la ecuación:

$$
\text { NUS=Uso especie (s) x 100/ Nis }
$$

Donde: Uso especie (s): número de citaciones para cada especie; Nis: número de informantes encuestados.

- Índice de fidelidad: Se calculó de acuerdo a la siguiente fórmula:

$$
I F=\frac{F C C}{F T C} \times 100
$$


Donde: FCC es la frecuencia de citación en la categoría y FTC es la frecuencia total de citaciones.

Esta fórmula se aplicó para comparar los datos de los diferentes usos de la planta donde la encuesta fue aplicada.

Índice de consenso entre los informantes: Fue calculado según la siguiente ecuación:

$$
F C=\frac{(F C C-N T C)}{(F C C-1)}
$$

Donde FCC es la frecuencia de citación en cada categoría y NTC el número total de especies en la categoría.

Cuando este valor es igual a 1 es un indicador de que existe consenso en la población acerca de la utilidad de las plantas que han sido informadas (Laffita et al, 2020).

\section{Resultados y discusión}

\section{Características de la muestra de estudio}

\section{Descripción sociodemográfica}

Se realizaron un total de 100 entrevistas en los municipios de Santiago de Cuba y Songo La Maya, 50 en cada caso. El sexo predominante fue el femenino (69\% del total de encuestados), y, a pesar de que en el caso particular del municipio Santiago de Cuba, fue realizado igual número de entrevistas tanto a hombres como a mujeres, fueron ellas las que refirieron y demostraron un mayor conocimiento en el uso de plantas medicinales. Esto se debe a que, por lo general, en la población cubana, es la mujer quien tiene una mayor responsabilidad con tareas relacionadas con la salud familiar. Los informantes fueron divididos en tres grupos etáreos: el primero de 16-39 años, el segundo de 40-59 años y el tercero de más de 60 años. En el municipio Songo La Maya el grupo etáreo predominante comprendió el rango entre 40-59 años (64\%), mientras que para Santiago de Cuba fue el de 60 años o más (51\%). Este último aspecto pudiera estar relacionado al hecho de que los individuos, en la medida que envejecen, aparecen con más frecuencia las enfermedades crónicas como la hipertensión arterial y diabetes, reforzando las tradiciones empíricas que han observado y de las que han participado a lo largo de toda su vida. Por esta razón, probablemente, adquieren un mayor conocimiento de los modos de curar y en especial, del uso tradicional de las plantas medicinales.

En lo referente al nivel de instrucción y ocupación los resultados fueron los siguientes: En Santiago de Cuba predominaron los jubilados y las amas de casa, seguidos de obreros, universitarios y estudiantes. De los trabajadores, la mayoría trabaja para el sector estatal. De los encuestados de la localidad de Songo La Maya, predominó el egresado universitario, seguido de técnicos medios, jubilados y estudiantes. Treinta de ellos se encuentran activos laboralmente, dos amas de casa y 18 son jubilados. En sentido general se observa que abundan usuarios de la medicina tradicional que se encuentran 
desvinculados de la vida laboral por la edad que presentan, pero el uso de las plantas medicinales como vía alternativa de sanación no demuestra ni relación con el nivel de instrucción ni de ocupación; demostrando que es una práctica establecida y común entre la población de las dos localidades estudiadas. Tampoco se encontró relación lineal directa entre el empleo de plantas y el tipo de población urbana o suburbana.

Del total de pacientes entrevistados en Santiago de Cuba, 25 padecía de hipertensión arterial; seis de diabetes mellitus tipo II; nueve con estos dos padecimientos, y diez pacientes con asma bronquial. En Songo La Maya 23 pacientes padecen de asma bronquial, 21 pacientes de hipertensión arterial y 6 pacientes de diabetes mellitus (ver Figura 1).

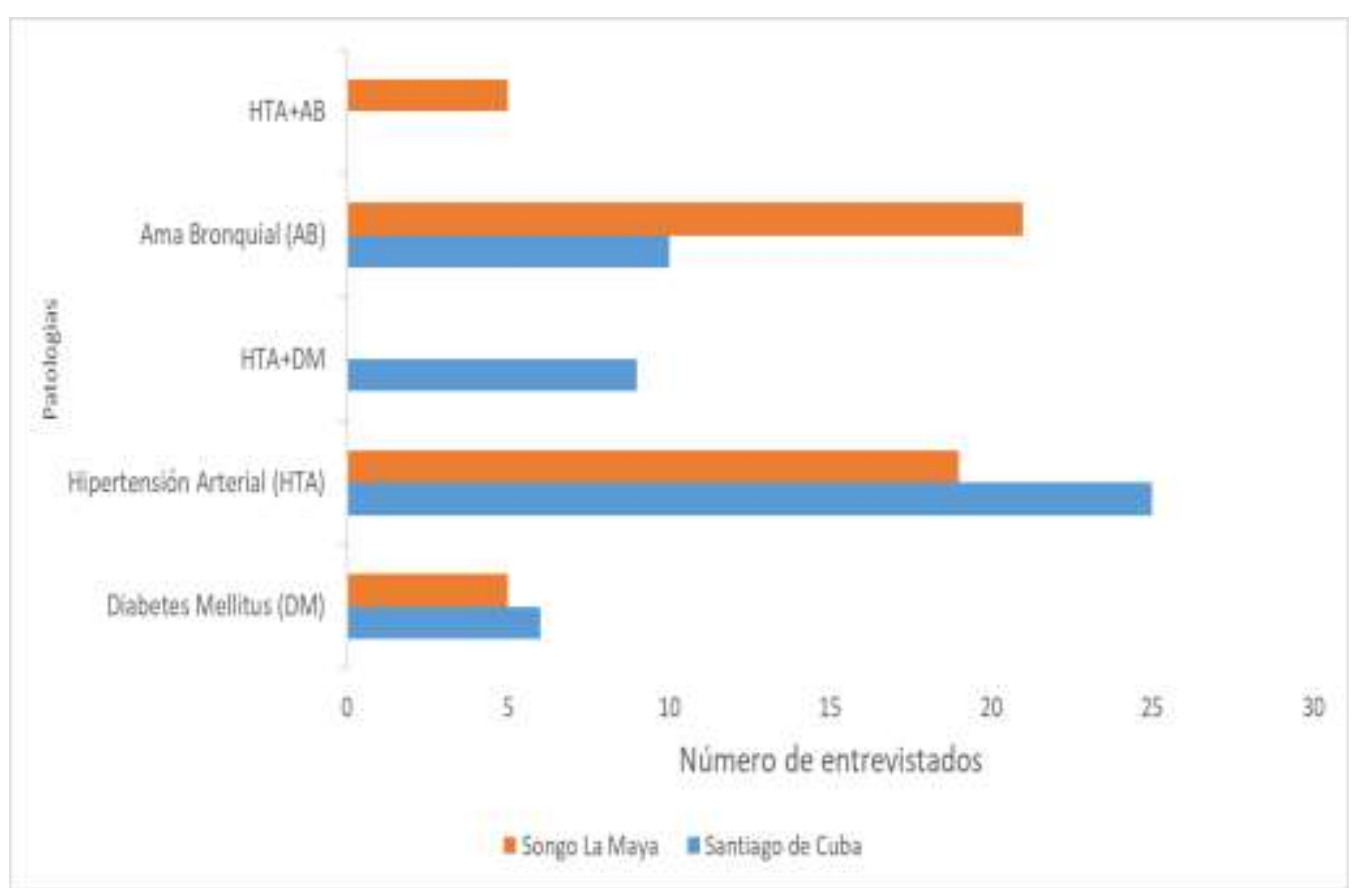

Figura 1. Enfermedades crónicas no transmisibles asociadas al empleo de plantas medicinales por la población encuestada.

Fuente: Elaborado por los autores

De la figura uno se observa que a pesar de que la proporción de pacientes hipertensos y diabéticos tipo II es más o menos la misma, en la población urbana de Santiago de Cuba es donde se observa la presencia de ambas patologías asociadas a un mismo paciente; indicando que probablemente la vida más dinámica y estresante de la gran ciudad exacerba la presencia de ambas comorbilidades. Como aspecto curioso, la mayor concentración de pobladores con asma bronquial se encuentra en la población suburbana; resaltando el hecho que a pesar de que el asma es una enfermedad que muchas veces viene asociada a la calidad medioambiental, no necesariamente depende de ello.

\section{Modos de preparación y uso de plantas medicinales}

Del total de las 100 encuestas realizadas se identificaron un total de 51 especies, agrupadas en 34 familias, empleadas en los municipios de Santiago de Cuba y Songo la 
Maya para el tratamiento de las enfermedades crónicas no transmisibles. Todas estas especies fueron identificadas taxonómicamente en el Centro de Oriental de Ecosistemas y Biodiversidad (BIOECO) en la provincia de Santiago de Cuba, lo cual da una mayor confiabilidad a los resultados obtenidos en este estudio. Un listado de las mismas, así como el destino de su empleo se relaciona en las tablas 1 y 2.

Tabla 1.

Plantas reportadas por la población de la comunidad de Santiago de Cuba para el tratamiento de las enfermedades crónicas no transmisibles.

\begin{tabular}{|c|c|c|c|c|c|c|}
\hline Familia & $\begin{array}{l}\text { Nombre } \\
\text { Científico }\end{array}$ & $\begin{array}{l}\text { Nombre } \\
\text { Común }\end{array}$ & $\begin{array}{c}\text { Parte } \\
\text { Utilizada }\end{array}$ & $\begin{array}{c}\text { Forma de } \\
\text { Preparación }\end{array}$ & Usos & Citas \\
\hline Liliaceae & $\begin{array}{l}\text { Allium } \\
\text { sativum L. }\end{array}$ & Ajo & $\begin{array}{l}\text { Dientes/ } \\
\text { (bulbos) }\end{array}$ & Decocción & Antihipertensivo & 24 \\
\hline Labiaceae & $\begin{array}{l}\text { Ocimum } \\
\text { gratissimum L. }\end{array}$ & $\begin{array}{l}\text { Albahaca } \\
\text { de clavo }\end{array}$ & Hojas & Infusión & Diurético & 24 \\
\hline Rutaceae & $\begin{array}{l}\text { Citrus } \\
\text { aurantifolia }\end{array}$ & Limón & $\begin{array}{l}\text { Hoja/ } \\
\text { cáscara }\end{array}$ & Decocción & Antihipertensivo & 20 \\
\hline Mirtaceae & $\begin{array}{l}\text { Psidium } \\
\text { guajaba L. }\end{array}$ & Guayaba & Hojas & Infusión & Antihipertensivo & 20 \\
\hline Lauraceae & $\begin{array}{l}\text { Persea } \\
\text { americana }\end{array}$ & Aguacate & $\begin{array}{l}\text { Cáscara/ } \\
\text { semilla }\end{array}$ & Decocción & Diurético & 15 \\
\hline Anacardiacea & $\begin{array}{l}\text { Anacardium } \\
\text { occidentale } \mathrm{L} .\end{array}$ & Marañón & $\begin{array}{l}\text { Semilla/ } \\
\text { Corteza }\end{array}$ & Decocción & Diurético & 12 \\
\hline Cruciferaceae & $\begin{array}{l}\text { Nasturtium } \\
\text { officinale } \mathrm{R} . \\
\mathrm{Br}\end{array}$ & Berro & Hojas & Infusión & Diurético & 12 \\
\hline Labiaceae & $\begin{array}{l}\text { Salvia } \\
\text { officinalis L. }\end{array}$ & $\begin{array}{l}\text { Salvia de } \\
\text { castilla }\end{array}$ & Hojas & Infusión & Diurético & 11 \\
\hline Labiaceae & $\begin{array}{l}\text { Ocimum } \\
\text { sanctum L. }\end{array}$ & $\begin{array}{l}\text { Albahaca } \\
\text { morada }\end{array}$ & Hojas & infusión & Diabetes & 11 \\
\hline Asteraceae & $\begin{array}{l}\text { Chamaemelum } \\
\text { nobile L. }\end{array}$ & Camomila & $\begin{array}{l}\text { Hojas/ } \\
\text { flores }\end{array}$ & infusión & Broncodilatador & 9 \\
\hline Mirtáceae & $\begin{array}{l}\text { Eucalyptus } \\
\text { maculata }\end{array}$ & Eucalipto & Hojas & infusión & Expectorante & 9 \\
\hline Zingiberaceae & $\begin{array}{l}\text { Zingibe } \\
\text { rofficinalis } L .\end{array}$ & Jengibre & Rizomas & decocción & Expectorante & 8 \\
\hline Rutaceae & $\begin{array}{l}\text { Citrus } \\
\text { aurantifolia L. }\end{array}$ & Limón & Hojas & infusión & Broncodilatador & 8 \\
\hline Apiaceae & $\begin{array}{l}\text { Petroselinum } \\
\text { crispum Fuss }\end{array}$ & Perejil & Hojas & Infusión & Diurético & 8 \\
\hline Mirtaceae & $\begin{array}{l}\text { Eucalyptus } \\
\text { maculate } \mathrm{L}\end{array}$ & Eucalipto & Hojas & infusión & Diabetes & 8 \\
\hline
\end{tabular}


ORANGE JOURNAL

\begin{tabular}{|c|c|c|c|c|c|c|}
\hline Theaceae & $\begin{array}{l}\text { Camellia } \\
\text { sinensis L. }\end{array}$ & Té verde & Hojas & infusión & Diabetes & 7 \\
\hline Grossulainaceae & Ruva crispa $L$. & $\begin{array}{l}\text { Grosella } \\
\text { india }\end{array}$ & Hojas & infusión & Antiasmático & 4 \\
\hline Sapotaceae & $\begin{array}{l}\text { Pouteria } \\
\text { sapota L. }\end{array}$ & Zapote & Semilla & Decocción & Diurético & 4 \\
\hline Papilionaceae & $\begin{array}{l}\text { Trigonellafoen } \\
\text { um-graecum } \\
\text { L. }\end{array}$ & Alholva & Hojas & infusión & Diabetes & 3 \\
\hline Apocynaceae & $\begin{array}{l}\text { Gymnema } \\
\text { sylvestre }\end{array}$ & $\begin{array}{l}\text { Gymnema } \\
\text { sylvestre }\end{array}$ & Hojas & infusión & Diabetes & 3 \\
\hline Poaceae & $\begin{array}{l}\text { Cymbopogon } \\
\text { citratus Stapf }\end{array}$ & $\begin{array}{l}\text { Caña } \\
\text { Santa }\end{array}$ & Hojas & Infusión & Antihipertensivo & 3 \\
\hline Graminaceae & $\begin{array}{l}\text { Bambusa } \\
\text { vulgaris } \\
\text { Schrad. }\end{array}$ & $\begin{array}{l}\text { Caña } \\
\text { Brava }\end{array}$ & Hojas & Infusión & Diurético & 3 \\
\hline Labiadceae & $\begin{array}{l}\text { Mentha } \\
\text { arvensis L. }\end{array}$ & Menta & Hojas & infusión & Expectorante & 3 \\
\hline Apiaceae & $\begin{array}{l}\text { Pimpinella } \\
\text { anisum L. }\end{array}$ & Anís & Semilla & decocción & Antiasmático & 3 \\
\hline Lauraceae & Laurus nobilis & Laurel & Hojas & infusión & Antiasmático & 3 \\
\hline Sapindaceae & $\begin{array}{l}\text { Allophylus } \\
\text { cominia (L.) } \\
\text { Sw. }\end{array}$ & $\begin{array}{l}\text { Palo de } \\
\text { caja }\end{array}$ & $\begin{array}{l}\text { Ramas/ } \\
\text { raíces }\end{array}$ & decocción & Antidiabético & 1 \\
\hline
\end{tabular}

Fuente: Elaborado por los autores

Tabla 2.

Plantas reportadas por la población de la comunidad de Songo la Maya para el tratamiento de las enfermedades crónicas no transmisibles.

\begin{tabular}{|c|c|c|c|c|c|c|}
\hline Familia & $\begin{array}{l}\text { Nombre } \\
\text { Científico }\end{array}$ & $\begin{array}{l}\text { Nombre } \\
\text { Común }\end{array}$ & $\begin{array}{l}\text { Parte } \\
\text { Utilizada }\end{array}$ & $\begin{array}{l}\text { Forma de } \\
\text { Preparación }\end{array}$ & Usos & Citas \\
\hline Annonaceae & $\begin{array}{l}\text { Annona } \\
\text { muricata L. }\end{array}$ & Guanábana & Fruto & zumo & $\begin{array}{l}\text { Antiasmático, } \\
\text { Antihipertensivo }\end{array}$ & 32 \\
\hline Rutaceae & $\begin{array}{l}\text { Citrus } \\
\text { aurantium L }\end{array}$ & Naranja agria & Fruto & zumo & $\begin{array}{l}\text { Antiasmático, } \\
\text { Antihipertensivo }\end{array}$ & 32 \\
\hline Rutaceae & $\begin{array}{l}\text { Citrus } \\
\text { reticulate } \mathrm{L}\end{array}$ & Mandarina & Cascara & decocción & Antiasmático & 22 \\
\hline Verbenaceae & $\begin{array}{l}\text { Verbena } \\
\text { officinalis L. }\end{array}$ & Verbena & Hojas & decocción & $\begin{array}{l}\text { Antiasmático, } \\
\text { Antihipertensivo }\end{array}$ & 22 \\
\hline Asteraceae & $\begin{array}{l}\text { Calendula } \\
\text { officinalis L. }\end{array}$ & Caléndula & Hojas & infusión & Antihipertensivo & 21 \\
\hline Caricaceae & $\begin{array}{l}\text { Carica } \\
\text { papaya } \mathrm{L} .\end{array}$ & Fruta bomba & Hojas & decocción & Antihipertensivo & 21 \\
\hline Malvaceae & $\begin{array}{l}\text { Gossypium } \\
\text { barbadensis } \\
\mathrm{L}\end{array}$ & Algodón & Hojas & decocción & Antiasmático & 20 \\
\hline Phytolacaceae & $\begin{array}{l}\text { Petiveria } \\
\text { alliacea } \mathrm{L} \text {. }\end{array}$ & Anamú & Hojas & infusión & Antiasmático & 20 \\
\hline
\end{tabular}


ORANGE JOURNAL

\begin{tabular}{|c|c|c|c|c|c|c|}
\hline Boraginaceae & $\begin{array}{l}\text { Cordia } \\
\text { collocca } \mathrm{L} .\end{array}$ & Ateje & Hojas & infusión & Antihipertensivo & 20 \\
\hline Rhamnaceae & $\begin{array}{l}\text { Colubrina } \\
\text { elliptica } \\
\text { Brizicky. }\end{array}$ & Bijagua & Hojas & infusión & Antiasmático & 20 \\
\hline Rutaceae & $\begin{array}{l}\text { Citrus } \\
\text { paradise } \mathrm{L} .\end{array}$ & Toronja & Hojas & decocción & $\begin{array}{l}\text { Antiasmático, } \\
\text { Antihipertensivo }\end{array}$ & 20 \\
\hline Combretaceae & $\begin{array}{l}\text { Terminalia } \\
\text { catappa } \\
\text { Sarg }\end{array}$ & Almendra & Hojas & infusión & Antihipertensivo & 19 \\
\hline Lamiaceae & $\begin{array}{l}\text { Mentha } \\
\text { citrate Ehrb }\end{array}$ & Toronjil & Hojas & decocción & Antihipertensivo & 18 \\
\hline Lamiaceae & $\begin{array}{l}\text { Plectranthus } \\
\text { neochillus } \\
\text { Schltl }\end{array}$ & Meprobamato & Hojas & decocción & Antihipertensivo & 17 \\
\hline Urticaceae & $\begin{array}{l}\text { Cecropia } \\
\text { peltata } \mathrm{L} .\end{array}$ & Yagruma & Hojas & decocción & Antiasmático & 17 \\
\hline Apiaceae & $\begin{array}{l}\text { Petroselinu } \\
\text { m crispum } \\
\text { Fuss }\end{array}$ & Perejil & Hojas & infusión & Antihipertensivo & 16 \\
\hline Poaceae & $\begin{array}{l}\text { Cymbopogo } \\
n \text { citratus } \\
\text { Stapf }\end{array}$ & Cañasanta & Hojas & infusión & Antiasmático & 15 \\
\hline Lamiaceae & $\begin{array}{l}\text { Majorana } \\
\text { hortensis } \\
\text { (Moch) }\end{array}$ & Mejorana & Hojas & decocción & Antiasmático & 13 \\
\hline Solanaceae & $\begin{array}{l}\text { Solanum } \\
\text { nigrum L. } \\
\text { var. } \\
\text { americanum } \\
\text { (Mill) } \\
\text { Schuiz }\end{array}$ & Yerba mora & Hojas & decocción & Antihipertensivo & 13 \\
\hline Amaryllidaceae & $\begin{array}{l}\text { Allium cepa } \\
\text { L. }\end{array}$ & Cebolla & Hojas & decocción & Antiasmático & 12 \\
\hline Commelinaceae & $\begin{array}{l}\text { Rhoeo } \\
\text { discolor } \\
\text { Hance }\end{array}$ & Cordobán & Hojas & decocción & $\begin{array}{l}\text { Antiasmático, } \\
\text { Antihipertensivo }\end{array}$ & 12 \\
\hline Bignonaceae & $\begin{array}{l}\text { Crescentia } \\
\text { cujete L. }\end{array}$ & Güira & Hojas & infusión & Antiasmático & 10 \\
\hline Apiaceae & $\begin{array}{l}\text { Foeniculum } \\
\text { vulgare Mill }\end{array}$ & Hinojo & Hojas & decocción & Antiasmático & 10 \\
\hline Meliaceae & Melia sp. & Lila & Hojas & infusión & Antiasmático & 9 \\
\hline Asteraceae & $\begin{array}{l}\text { Bidens alba } \\
\text { Lin. }\end{array}$ & Romerillo & Hojas & infusión & Antiasmático & 8 \\
\hline Fabaceae & $\begin{array}{l}\text { Dichrostach } \\
\text { ys cinerea }\end{array}$ & Marabú & Hojas & infusión & Diabetes & 5 \\
\hline Anacardiaceae & $\begin{array}{l}\text { Mangifera } \\
\text { indica L. }\end{array}$ & Mango & Hojas & decocción & Diabetes & 4 \\
\hline Asteraceae & $\begin{array}{l}\text { Helianthus } \\
\text { annuus L. }\end{array}$ & Girasol & Hojas & infusión & Antihipertensivo & 4 \\
\hline Arecaceae & $\begin{array}{l}\text { Cocos } \\
\text { nucifera L. }\end{array}$ & Coco & Fruto & agua de coco & Diurético & 3 \\
\hline
\end{tabular}

Fuente: Elaborado por los autores. 
De las tablas 1 y 2 se infiere que la población de la comunidad rural hace un uso más extensivo de las plantas medicinales que la población urbana, no sólo por el número total de especies empleadas (29 vs 26), sino también por el número total de citas (455 vs 236). Resulta curioso además que de las plantas citadas en ambas localidades cuatro especies solamente resultan coincidentes (Perejil, Eucalipto, Caña Santa y Limón), pero más aún el hecho de que sólo para el Perejil la actividad informada es la misma. Estas observaciones pueden deberse al hecho de que en las zonas rurales existe una mayor biodiversidad, y con ello mayor disponibilidad de especies para su utilización. Es internacionalmente aceptado que en las comunidades rurales el empleo de los recursos de la naturaleza por parte de sus habitantes es muy superior a los de la zona urbana, algo que ha sido informado también para Cuba (Heredia et al, 2018)

La parte de la planta con una mayor utilización por la población de ambas localidades fueron las hojas, representando el $96 \%$ del total de reportes. Estas observaciones probablemente, tienen que ver, con que son la parte más asequible de la planta, que tienen un mejor manejo y un extenso historial de empleo, algo que coincide con estudios etnobotánicos anteriores en la población cubana (Pérez et al., 2011; Heredia et al, 2018). Le sigue en orden de frecuencia los frutos y la raíz, partes de las plantas también muy empleadas por la población para el tratamiento de las enfermedades objeto de estudio ya que en las mismas también aparece un gran número de metabolitos secundarios muy útiles en el tratamiento de estas patologías (Llauradó et al., 2020). La planta fresca como forma de uso fue predominante en la población encuestada con $89 \%$ del total de reportes, los pacientes refieren que solamente recolectan la planta cuando va a ser utilizada de manera inmediata o en el transcurso del día. De las vías de administración, la oral resultó ser la más empleada por los encuestados, con un $97 \%$ del total de reportes. La vía oral resulta la ruta natural de entrada de alimentos y medicamentos al organismo, es la menos invasiva y para la cual los métodos de preparación son más sencillos.

En la figura 2, se representan las formas de preparación más empleadas por los encuestados, siendo la infusión la más empleada con un total de $66 \%$ del total de reportes, le siguen en orden decreciente la decocción con 23 citaciones y en menor medida el zumo con 11 citaciones.

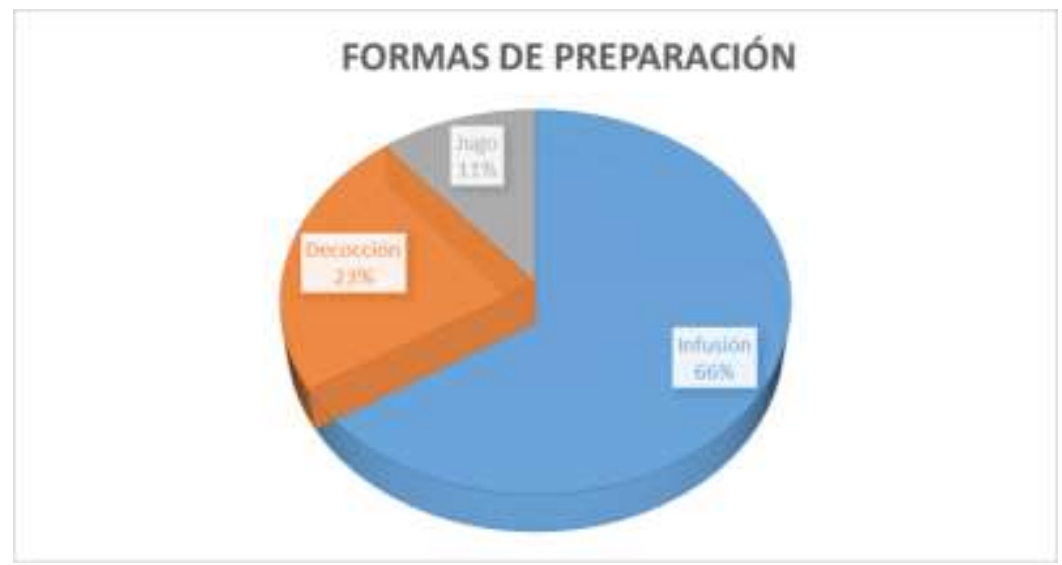

Figura 2. Formas de preparación más empleadas por la población.

Fuente: Elaborado por los autores. 
De manera general la cantidad que se administra de la preparación lo realizan de forma empírica sin una unidad de medida establecida, refiriendo la cucharada en caso de que la preparación fuera el zumo y el vaso en el caso de la infusión y la decocción. La totalidad de los encuestados plantean que usan la preparación vegetal por el tiempo que dure la enfermedad, independientemente de la forma de preparación. El período más empleado a la hora de la colección de las plantas es el vegetativo con $94 \%$ del total de reportes.

\section{Clasificación de las especies según patología a emplear}

\section{Hipertensión Arterial:}

En la localidad de Santiago de Cuba, un total de nueve especies se emplean para disminuir la tensión arterial, mientras que en la localidad e Songo la Maya asciende a 15, cumpliéndose la observación de que en la comunidad rural es mayor el número de plantas empleadas, aún cuando esta patología tiene mayor incidencia en la población urbana (ver figura 1). El uso de plantas medicinales entre ambas localidades para el tratamiento de esta patología representa el 47,05\% del total de plantas informadas ( 24 especies de 51 en total)

Es notorio además el hecho de que la población urbana hace una diferenciación más certera entre el valor diurético y antihipertensivo de la especie, aún cuando es claro que la diuresis es una de los mecanismos fisiológicos para disminuir la tensión arterial. Este conocimiento más específico, no está relacionado el nivel de instrucción, pues como se enunció anteriormente no existían diferencias entre los niveles de escolaridad de los entrevistados en ambas localidades.

\section{Diabetes mellitus}

Para esta patología, el número de especies citadas es superior en los pobladores de la comunidad urbana (seis) que en los de la comunidad rural (dos), aún cuando no hay gran diferencia entre el número de encuestados que padecen de esta enfermedad (ver figura 1). Los hábitos alimentarios más sanos y el propio modo de vida menos estresante pueden ser los factores que condicionan estos hallazgos. Entre ambas localidades el número de plantas empleadas representan solamente el 15,68\% del total de las empleadas.

\section{Asma bronquial}

De las 29 especies medicinales informadas por la población de la comunidad rural de Songo la Maya, 17 se emplean para el tratamiento del asma. De igual manera y a pesar que el grado de incidencia del asma bronquial en los pobladores de la comunidad urbana de Santiago de Cuba fue mucho más bajo que en la comunidad rural, el número de especies es también elevado (ocho). Entre ambas localidades aportan 25 de las 51 especies informadas, representando casi el 50\% de las mismas. Ello podría ilustrar la naturaleza de esta enfermedad, la cual es multicasual y de difícil control. Nuevamente, los informantes de la localidad urbana resultan más específico en su definición de actividad al clasificarlas mayormente por su mecanismo de acción (broncodilatador, expectorante... etc). 
Un aspecto común en ambas áreas de estudio resultó el uso empírico de estas plantas. Los entrevistados aceptaban sugerencias de familiares y amigos basados en sus experiencias, sin embargo, no consultaban al facultativo ni al farmacéutico a fin de conocer si verdaderamente esa especie vegetal que se estaban administrando tenía probado efecto medicinal para esa patología. En la inmensa mayoría de los casos, los pacientes entrevistados habían abandonado por completo su esquema de medicación y lo habían sustituido por este tratamiento "no convencional", y los que se mantuvieron consumiendo sus medicamentos se lo administraban concomitantemente con la planta, sin percatarse de los riesgos que pueden ocurrir en caso tal que una especie potencie o inhiba el efecto farmacológico.

\section{Indicadores cuantitativos del estudio realizado}

\section{Índice de valor de uso (IVU) y nivel de uso significativo (NUS)}

Una de las principales finalidades de las investigaciones etnobotánicas es encontrar especies medicinales con potencial para validar su uso etnofarmacológico. A partir de esta información las plantas pueden estudiarse con mayor detalle y lograr aislar metabolitos activos importantes o sencillamente obtener preparados a base de la planta, utilizando como principio activo los extractos estandarizados de la misma.

En este sentido, los indicadores IVU y NUS expresan, que aquellos usos medicinales que son citados con una frecuencia superior o igual a un $20 \%$ por las personas encuestadas que usan plantas como primer recurso para un determinado problema de salud, pueden considerarse significativos desde el punto de vista de su aceptación cultural y por lo tanto merecen su evaluación y validación científica.

De las 51 especies de plantas medicinales informadas, un total de 28 cumplen con un NUS igual o superior al $20 \%$, es por ello que en la tabla 3 sólo se muestran las plantas medicinales con IVU y NUS superiores a 0,4 y $40 \%$ respectivamente. El ajo y la albahaca clavo clasificaron como las de mayores índices y curiosamente corresponden a especies informadas por habitantes de la localidad urbana que fueron los que informaron en menor grado el uso de plantas. Ello realza la naturaleza de la información que ofrecen estos índices al descontextualizar el origen de la información y centrarlo en las especies empleadas. Esta tabla demuestra además una total coincidencia con la naturaleza y frecuencia de las enfermedades crónicas que padecen los encuestados y expresadas en la figura 1, pues el número de especies y los índices IVU y NUS coinciden en orden con los tratamientos alternativos que utiliza la población para la hipertensión y el asma. Las restantes especies no incluidas en la tabla y que sobrepasan un NUS superior al 20\% son: Almendra, Toronjil, Meprobamato, Yagruma, Perejil, Aguacate, Caña Santa, Yerba mora, Mejorana, Berro, Marañón, Cebolla, Albahaca morada, Salvia de castilla, Güira y el Hinojo. 
Tabla 3.

Índice de valor de uso y nivel de Uso significativo de las plantas más reportadas para cada enfermedad.

\begin{tabular}{|l|l|l|l|l|l|}
\hline Nombre Científico & $\begin{array}{l}\text { Nombre } \\
\text { Común }\end{array}$ & Citas & IVU & NUS & Usos \\
\hline Allium sativum L. & Ajo & 24 & 0,48 & 48 & Antihipertensivo \\
\hline Ocimum gratissimum L. & $\begin{array}{l}\text { Albahaca de } \\
\text { clavo }\end{array}$ & 24 & 0,48 & 48 & $\begin{array}{c}\text { Antihipertensivo } \\
\text { (Diurético) }\end{array}$ \\
\hline Citrus reticulate L. & Mandarina & 22 & 0,44 & 44 & Antiasmático \\
\hline Calendula officinalis $L$ & Caléndula & 21 & 0,42 & 42 & Antihipertensivo \\
\hline Carica papaya L. & Fruta bomba & 21 & 0,42 & 42 & Antihipertensivo \\
\hline Psidium guajaba L. & Guayaba & 20 & 0,40 & 40 & Antihipertensivo \\
\hline Citrus aurantifolia L. & Limón & 20 & 0,40 & 40 & Antihipertensivo \\
\hline Cordia collocca L. & Ateje & 20 & 0,40 & 40 & Antihipertensivo \\
\hline $\begin{array}{l}\text { Gossypium barbadensis } \text { L. } \\
\text { Petiveria alliacea } \text { L. }\end{array}$ & Algodón & 20 & 0,40 & 40 & Antiasmático \\
\hline $\begin{array}{l}\text { Colubrina } \text { elliptica } \\
\text { Brizicky. }\end{array}$ & Anamú & 20 & 0,40 & 40 & Antiasmático \\
\hline
\end{tabular}

Fuente: Elaborado por los autores

Índice de fidelidad (IF)

El índice de fidelidad (IF) es una expresión cuantitativa no solo de aceptación sino también de la confianza de las personas en el uso de una especie específica con una finalidad farmacológica determinada. El valor de índice de fidelidad es un indicador que señala la proporción de los informantes que utilizó una especie determinada para un uso en particular (FCC), con relación al total de informantes que reportaron usar plantas para ese uso (FTC). La tabla 4 recoge los valores obtenidos para las especies con un IF igual o mayor al $80 \%$

Tabla 4.

Usos de plantas medicinales y su Índice de fidelidad para el tratamiento de enfermedades crónicas no transmisibles.

\begin{tabular}{|c|c|c|c|c|c|}
\hline Nombre Científico & Nombre Común & FCC & FTC & IF & Uso \\
\hline Calendula officinalis $L$ & Caléndula & 21 & 21 & 100 & Antihipertensivo \\
\hline Carica papaya $\mathbf{L}$. & Fruta bomba & 21 & 21 & 100 & Antihipertensivo \\
\hline Allium sativum $\mathbf{L}$. & Ajo & 24 & 25 & 96,00 & Antihipertensivo \\
\hline Ocimum gratissimum $\mathbf{L}$. & Albahaca de clavo & 24 & 25 & 96,00 & $\begin{array}{c}\text { Antihipertensivo } \\
\text { (Diurético) }\end{array}$ \\
\hline Citrus reticulate $\mathbf{L}$ & Mandarina & 22 & 23 & 95,65 & Antiasmático \\
\hline
\end{tabular}


ORANGE JOURNAL

\begin{tabular}{|l|l|c|c|c|c|}
\hline Cordia collocca L. & Ateje & 21 & 22 & 95,24 & Antihipertensivo \\
\hline Terminalia catappa Sarg & Almendra & 19 & 21 & 90,48 & Antihipertensivo \\
\hline Chamaemelum nobile L. & Camomila & 9 & 10 & 90,00 & Antiasmático \\
\hline Gossypium barbadensis L. & Algodón & 20 & 23 & 86,95 & Antiasmático \\
\hline Petiveria alliacea L. & Anamú & 20 & 23 & 86,95 & Antiasmático \\
\hline Colubrina elliptica Brizicky. & Bijagua & 20 & 23 & 86,95 & Antiasmático \\
\hline $\begin{array}{l}\text { Mentha citrate Ehrb } \\
\text { Plectranthus neochillus }\end{array}$ & Toronjil & 18 & 21 & 85,71 & Antihipertensivo \\
\hline $\begin{array}{l}\text { Schltl } \\
\text { Psidium guajaba } \text { L. }\end{array}$ & Guayaba & 20 & 25 & 80,00 & Antihipertensivo \\
\hline
\end{tabular}

Fuente: Elaborado por los autores

Como se puede observar, para el caso de la caléndula y la frutabomba, todos los informantes que declararon el uso de estas especies lo hicieron sobre la base de su uso como antihipertensivos, alcanzando un consenso total. La validez del empleo de este índice puede ilustrarse con el resultado alcanzado por la camomila, una especie relativamente poco informada, pero con elevado consenso poblacional en su uso como antiasmático. No obstante, nuevamente predominan las plantas empleadas para el tratamiento de la hipertensión arterial y el asma bronquial.

La profundización en el estudio farmacológico y de composición fitoquímica de estas especies (principalmente las señaladas en las tablas 3 y 4) se podrían obtener evidencias científicas que contribuyan a validar el uso etnobotánico de estas plantas. En el caso de que no existan reportes al respecto, sería entonces una buena oportunidad para continuar el estudio de estas especies con potencial terapéutico. Sin embargo, a la razón de este trabajo, estos detalles quedarán como premisas para nuevas investigaciones.

\section{Conclusiones}

Se informa un total de 51 especies, agrupadas en 34 familias, informadas por pobladores con enfermedades crónicas no transmisibles pertenecientes a una localidad urbana y otra rural de la provincia de Santiago de Cuba. Se observan diferencias entre el empleo de las plantas medicinales entre ambas localidades y se resaltan como datos de interés aquellas especies con mayor índice de valor de uso (IVU), nivel de uso significativo (NUS) e índice de fidelidad, siendo las de mayor potencial etnobotánico el Ajo, la Albahaca de clavo, la Caléndula y la Fruta bomba, según el índice valorado. Se describen además los principales datos sociodemográficos y clínicos de cada zona, resaltando como enfermedad crónica no transmisible más común la Hipertensión arterial y en consecuencia, el número total de plantas empleadas para su tratamiento. 
Referencias bibliográficas

Badke, M. R., Budó, M. D. L. D., Alvim, N. A. T., Zanetti, G. D., \& Heisler, E. V. (2012). Saberes y prácticas populares en el cuidado de la salud con el uso de plantas medicinales. Texto \& Contexto-Enfermagem, 21(2), 363-370.

Agarwal, R., Dhooria, S., Aggarwal, A. N., Maturu, V. N., Sehgal, I. S., Muthu, V., ... \& Varma, S. (2015). Guidelines for diagnosis and management of bronchial asthma: Joint ICS/NCCP (I) recommendations. Lung India: official organ of Indian Chest Society, 32(Suppl 1), S3.

Chávez, C. A., Hernández, C. A., García, I., Díaz, M. D., \& Tisoy, H. M. (2016). Las enfermedades no transmisibles como una de las enfermedades de interés para la salud pública. (Diplomado de profundización en fundamentos de Salud Pública). Universidad Nacional y a Distancia (UNAD), Colombia. https://repository.unad.edu.co/handle/10596/17550

Escalona, L. J., Tase, A., Estrada, A., \& Almaguer, M. L. (2015). Uso tradicional de plantas medicinales por el adulto mayor en la comunidad serrana de Corralillo Arriba. Guisa, Granma. Revista Cubana de Plantas Medicinales, 20(4).

Fernández, B. E., \& Armas, R. C. (2013). Producción científica cubana sobre plantas medicinales y productos naturales a partir de la base de datos PlantMedCUBA, 1967-2010. Revista Cubana de Plantas Medicinales, 18(3), 348-360

Hashmi, N. R., \& Khan, S. A. (2016). Adherence to diabetes mellitus treatment guidelines from theory to practice: the missing link. Journal of Ayub Medical College Abbottabad, 28(4), 802-808.

Heredia-Díaz, Y., García-Díaz, J., López-González, T., Chil-Nuñez, I., Arias-Ramos, D., Escalona-Arranz, J. C., ... \& Martínez-Figueredo, Y. (2018). An ethnobotanical survey of medicinal plants used by inhabitants of Holguín, Eastern Region, Cuba. Boletín latinoamericano y del caribe de plantas medicinales y aromáticas, 17(2)

Herrera, M. K. (2017). Eficacia del seguimiento farmacoterapéutico en pacientes hipertensos atendidos en la botica Rodrifarma del distrito El Porvenir-Trujillo. Octubre 2017-enero 2018 (Tesis para optar por el título profesional de Químico Farmacéutico). Universidad Católica de Los Ángeles Chimbote, Trujillo, Perú.

Intriago, L. F. Z., Allauca, M. P. B., Rodríguez, N. J. M., \& Romero, E. J. (2015). Estudio etnobotánico de plantas medicinales utilizadas por los habitantes del área rural de la Parroquia San Carlos, Quevedo, Ecuador. Universidad y Salud, 17(1), 97-111.

Laffita, I. U., Rodríguez, L. P., Naranjo, E. T., \& Hernández, Y. L. I. (2020). Caracterización etnobotánica de la Lawsonia inermis L. en el Distrito José Martí Norte, Santiago de Cuba. Revista Científica del Amazonas, 3(6), 6-17, https://revistadelamazonas.info/index.php/amazonas/article/view/35

Luján, M. C. (2015). Caracterización etnobotánica de las prácticas de medicina humana y veterinaria en poblaciones rurales, suburbanas y urbanas de Córdoba (Tesis de Doctorado en Ciencias Biológicas). Universidad Nacional de Córdoba, Córdoba, Argentina.

Maury, G. L., Rodríguez, D. M., Hendrix, S., Arranz, J., Boix, Y. F., Pacheco, A. O., Díaz, J. G., Morris-Quevedo, H. J., Dubois, A. F., Aleman, E. I., Beenaerts, N., Méndez-Santos, I. E., Ratón, T. O., Cos, P., \& Cuypers, A. (2020). Antioxidants in Plants: A Valorization Potential Emphasizing the Need for the Conservation of 
Plant Biodiversity in Cuba. Antioxidants (Basel, Switzerland), 9(11), 1048. https://doi.org/10.3390/antiox9111048

Mora, G. R., Verdecia, K., Rodríguez, T. D. L. M., del Pino, B. N., \& Guerra, C. (2017). Adherencia terapéutica en pacientes con algunas enfermedades crónicas no transmisibles. Revista Cubana de Medicina General Integral, 33(3), 270-280.

Pérez, M., Morón, F., Sueiro, M. L., Boffill, M., Lorenzo, G., Méndez, O. R., \& Blanco, F. (2011). Validación etnofarmacológica de Nectandracoriacea (Sw.) Griseb. y Caesalpiniabahamensis Lam. reportadas como diuréticas en el municipio Santa Clara. Revista Cubana de Plantas Medicinales, 16(2), 115-134.

Rodríguez, R. G., \& García, J. C. (2018). Comportamiento de las enfermedades crónicas no transmisibles en adultos mayores. Revista de Enfermedades no Transmisibles Finlay, 8(2), 103-110.

Rodríguez-Ferreiro, A.O., Léon-Duharte, D., Polanco-Durán, G., Guisado-Bourzac, F., Ochoa-Pacheco, A., \& Escalona-Arranz, J.C. (2020). Ethnobotany of Plectranthus neochilus Schltr (Meprobamate) in Cuba. Boletín latinoamericano y del caribe de plantas medicinales y aromáticas, 19 (2), 236-246

Roig, J. T. (1988). Diccionario Botánico de nombres vulgares cubanos. 3th edition, La Habana, Edt Nacional de Cuba.

Roig, J. T., (1974). Plantas medicinales, aromáticas o venenosas en Cuba. La Habana: Editorial Científico-técnica.

Soca, M.P., Sarmiento, Y., Mariño, A., Llorente, Y., Rodríguez, T., \& Peña, M. (2017). Prevalencia de enfermedades crónicas no transmisibles y factores de riesgo en adultos mayores de Holguín. Revista Finlay, 7(3), 155-167. 